\title{
44. SOBRE LA POSICIÓN FITOSOCIOLÓGICA DE FUMANA JUNIPERINA (CISTACEAE) EN EL SO DE LA PENÍNSULA IBÉRICA
}

\author{
Íñigo SÁNCHEZ GARCÍA y Antonio GALÁN DE MERA
}

About the phytosociological position of Fumana juniperina (Cistaceae) in the SW of the Iberian Peninsula.

Palabras clave. Fumana juniperina, fitosociología, España.

Key Words. Fumana juniperina, phytosociology, Spain.

Fumana juniperina (Lag. ex Dunal) Pau es un taxón mediterráneo occidental [España, Francia, Italia, Marruecos, Túnez](Willkomm in Willkomm \& Lange, 1880; Sampaio, 1947; Molero \& Rovira, 1987) del que hasta el momento existían escasos datos de herborización (Güemes \& Molero, 1993).

Los estudios de flora y vegetación que estamos realizando en la costa gaditana han revelado nuevas localidades y también datos más precisos sobre sus preferencias por sustratos areno-arcillosos guijarrosos, características ecológicas coincidentes con las descritas por Sauvage (1961) en Marruecos para F. laevis (Cav.) Pau.

En la costa de la provincia de Cádiz existen dos tipos de sustratos principales (Fontbote, 1987): arenas cuaternarias y arenas asociadas a arcillas pliocenas con un estrato superficial guijarroso. Sobre ambos tipos de sustratos existen grandes extensiones de pinares plagioclimácicos (Pinus pinea L.) que ocupan el dominio climácico de alcornocales y sabinares. Hasta la fecha, se han descrito en el SO de la Península Ibérica varias asociaciones del orden Stauracantho genistoidis-Halimietalia commutati RivasMartínez et al. 1990, cuyo significado ecológico es el de etapas subseriales de estos alcornocales y sabinares: Cistetum libanotidis Rothmaler 1954 (algarviense: algárvica), Thymo camphorati-Stauracanthetum spectabilis (Rothmaler 1954) Rivas-Martínez et al. 1990 (algarviense: promontorio vicentina, costero vicentina), Halimio commutatiCistetum libanotidis Rivas Goday 1955 (gaditano-onubense: onubense litoral), Halimio halimifolii-Stauracanthetum genistoidis RivasMartínez et al. 1980 (gaditano-onubense: onubense litoral), y Cisto salvifolii-Ulicetum australis Pérez Latorre et al. 1993 (hispalense, gaditano-onubense: gaditana litoral y aljíbica: sidonense, marbellí). La ausencia de elementos de estas asociaciones (Armeria macrophylla Boiss. \& Reuter, Armeria velutina Welw., Cistus libanotis L., Helichrysum picardii subsp. virescens (Valdés-Bermejo) Rivas-Martínez, Iberis ciliata subsp. welwitschii (Boiss.) Moreno, Lavandula sampaioana subsp. lusitanica (Chaytor) Rivas-Martínez, Díaz \& Fernández-González, Lavandula stoechas L., Pterocephalus intermedius (Lag.) Coutinho, Thymus albicans Hoffmanns. \& Link, Thymus albicans subsp. donyanae (Morales) RivasMartínez) en la unidad pliocena areno-arcillosa que se extiende entre las localidades de Conil de la Frontera y Chiclana de la Frontera (Cádiz), y la presencia de Eryngium dilatatum Lam., Festuca caerulescens Desf., Fumana juniperina (Lag. ex Dunal) Pau, Klasea monardii (Dufour) J. Holub, y Schoenus nigricans L. permiten describir una nueva asociación: Fumano juniperinae-Cistetum crispi ass. nova (typus: inv. 5, tabla 
Tabla 1

Fumano juniperinae-Cistetum crispi ass. nova. Typus inv. $\mathrm{n}^{\circ} 5$ (Coremion albi, Stauracantho-Halimietalia, Cisto-Lavanduletea)

$\mathrm{N}^{\circ}$ de orden

Altitud (m)

Superficie (m2)

$\mathrm{N}^{\circ}$ especies

$\begin{array}{rr}1 & 2 \\ 0 & 10 \\ 10 & 10 \\ 17 & 17\end{array}$

3

5

4

20

25

17

16

Características y diferenciales de asociación

Fumana juniperina

Cistus crispus

Eryngium dilatatum

Klasea monardii

Festuca caerulescens

$\begin{array}{lllll}1 & 1 & 1 & 2 & 2 \\ 2 & 2 & 1 & 1 & 1 \\ . & 2 & . & 3 & 2 \\ . & . & 1 & + & 1 \\ 1 & . & . & + & .\end{array}$

Características de Coremion albi, Stauracantho-Halimietalia y Cisto-Lavanduletea

Lavandula luisieri

Cistus salvifolius

Halimium halimifolium

Halimium commutatum

Ulex australis

Compañeras

Genista triacanthos

Pulicaria odora

Pistacia lentiscus

Chamaerops humilis

Schoenus nigricans

Linum bienne

Sanguisorba spachiana

Dipcadi serotinum

Calluna vulgaris

Rosmarinus officinalis

Biscutella microcarpa

Xolantha guttata

Centaurea africana

Teucrium fruticans

Taxones en un sólo inventario.- Inv. 1: Myrtus communis + , Polygala monspeliaca 1, Picris comosa 1, Juniperus turbinata + y Bellis perennis 1; Inv. 2: Avenula sulcata + y Margotia gummifera 1; Inv. 3: Urginea maritima + , Misopates grandiflorum + y Arenaria emarginata $+;$ Inv. 4: Tulipa australis 1, Juncus hybridus 4, Asphodelus ramosus 1, Margotia gummifera 1 y Cladonia sp. +.

LOCALIDADES: 1.- Pinar de San José, La Barrosa. Chiclana de la Frontera. Cádiz. 2.- Torre del Puerco. Chiclana de la Frontera. Cádiz. 3.- Faro de Roche. Conil de la Frontera. Cádiz. 4.- Pinar del Claverín. Chiclana de la Frontera. Cádiz. 5 (Typus).- Calas de Roche. Conil de la Frontera. Cádiz. 


\section{Coremion albi}

$\mathrm{N}^{\mathrm{o}}$ de inventarios

N. de orden

Características y diferenciales de asociaciones

Armeria velutina

Thymus donyanae

Cistus crispus.

Cistus monspeliensis

Genista hirsuta

Ulex australis

Fumana juniperina

Eryngium dilatatum

Klasea monardii

Festuca caerulescens

Características de Coremion albi

Cistus libanotis

Lavandula lusitanica

Corema album

Iberis welwitschii

Dianthus hinoxianus

Pterocephalus intermedius

Helichrysum virescens

Chifes

\author{
oremion albi
}

ca

18

1

1

1

4

10

5

$\begin{array}{llll}2 & 3 & 4 & 5\end{array}$

I

$\begin{array}{rrrr}\text { V } & \cdot & 4 & + \\ \text { V } & \cdot & \cdot & \cdot \\ \text { I } & \cdot & \cdot & \cdot \\ \text { I } & \cdot & \cdot & + \\ \text { III } & \cdot & \cdot & \cdot \\ . & \cdot & \cdot & + \\ \text { IV } & \cdot & \cdot & .\end{array}$

Características de Stauracantho-Halimietalia y Cisto-Lavanduletea

Halimium halimifolium

Halimium commutatum

Cistus salvifolius

Lavandula stoechas

Cistus ladanifer

Euphorbia baetica

Stauracanthus genistoides

Helichrysum serotinum

Thymus mastichina

Halimium viscosum

Lavandula luisieri

$\begin{array}{rrrrr}\text { V } & + & 4 & \text { V } & \text { II } \\ \text { V } & . & 4 & \text { IV } & \text { I } \\ . & . & 4 & \text { V } & \text { II } \\ . & . & 2 & \text { V } & . \\ . & . & 4 & . & . \\ . & . & . & \text { I } & . \\ \text { V } & . & . & \text { II } & . \\ . & . & . & . & . \\ . & . & 3 & . & . \\ . & . & 4 & . & \text { IV } \\ . & + & . & . & \text {. }\end{array}$

Procedencia de los inventarios. 1: Halimio halimifolii-Stauracanthetum genistoidis (Rivas-Martínez et al., 1980), tabla 58; 2: Comunidad de Cistus monspeliensis y C. crispus (Rivas-Martínez et al., 1980: 112); 3: Halimio commutati-Cistetum libanotidis (Rivas Goday, 1955), tabla 26; 4: Cisto salvifolii-Ulicetum australis (Pérez Latorre et al., 1993), tabla 11; 5: Fumano juniperinae-Cistetum crispi ass. nova.

Tabla 2. Tabla sintética de las asociaciones más próximas a Fumano juniperinae-Cistetum crispi.Synthetic table of the closest associations related to Fumano juniperinae-Cistetum crispi. 
1)[Coremion albi, Stauracantho-Halimietalia, Cisto-Lavanduletea] de areal gaditano litoral. Se trata de un matorral abierto que procede de la degradación de espinales de AsparagoRhamnetum oleoidis ericetosum scoparii Pérez Latorre et al. 1993, bajo ombroclima seco (Myrto-Querceto suberis halimietoso sigmetum). Por su ecología, es vicariante de la comunidad con Cistus monspeliensis L. y Cistus crispus L. que Rivas-Martínez et al. (1980) describieron en Doñana.

En la tabla 1, también destacan numerosos elementos de carácter atlántico mayoritariamente propios de la clase Calluno-Ulicetea $\mathrm{Br}$-B1. \& Tx. 1943 (Galán de Mera \& Vicente Orellana, 1996) que se introducen debido a la retención de humedad por las arcillas, como Calluna vulgaris (L.) Hull., Centaurea africana Lam., Genista triacanthos Brot. y Pulicaria odora (L.) Reichenb.Las diferencias florísticas de esta nueva asociación respecto a las comunidades más próximas quedan reflejadas en la tabla 2.

\section{BIBLIOGRAFÍA}

BRAUN-BLANQUET, J., A.R. PINTO DA SILVA y A. ROZEIRA -1964-Résultats de trois excursions géobotaniques à travers le Portugal septentrional et moyen. III. Landes à Cistes et Ericacées (Cisto-Lavanduletea et CallunoUlicetea). Agron. Lusit., 23 (4): 229-313.

GÜEMES, J. y J. MOLERO - 1993 - Fumana (Dunal) Spach. In: CASTROVIEJO, S., C. AEDO, S. CIRUJANO, M. LAINZ, P. MONTSERRAT, R. MORALES, F. MUÑOZ GARMENDIA, C. NAVARRO, J. PAIVA y C. SORIANO (eds.). Flora iberica. Madrid.

FONTBOTE, J.M. -1987- Mapa geológico de España. Cádiz. E 1: 200.000. IGME, Madrid.

GALÁN DE MERA, A. y J.A. VICENTE ORELLANA - 1996-Phy tosociological study of the plant communities with Stauracanthus boivinii of the SW of the Iberian Peninsula and NW of Africa, using multivariate analysis. Bot. Helv., 106: 45-56.
MOLERO, J. y A.M. ROVIRA -1987- Taxonomía del grupo Fumana thymifolia (Cistaceae). Candollea, 42: 501-531.

PÉREZ LATORRE, A.V., J.M. NIETO CALDERA y B. CABEZUDO -1993-Contribución al conocimiento de la vegetación de Andalucía. II. Los alcornocales. Acta Bot. Malacitana, 18: 223-258.

RIVAS GODAY, S. -1955- Aportaciones a la fitosociología hispánica. Anales Inst. Bot. Cavanilles, 13: 335-422.

RIVAS-MARTÍNEZ, S., M. COSTA, S. CASTROVIEJO y E. VALDÉS -1980Vegetación de Doñana. Lazaroa, 2: 5-189.

RIVAS-MARTÍNEZ, S., T.E. DÍAZ y F. FERNÁNDEZ-GONZÁLEZ -1990- Sobre la prioridad del nombre de la alianza Coremion albi (Stauracantho genistoidis-Halimion halimifolii). Itinera Geobot., 3: 127-130.

ROTHMALER, W. -1954- Vegetationsstudien in Nordwestspanien. Vegetatio, 5/6: 595-601.

SAMPAIO, G.A.-1947- Flora portuguesa. Oporto. SAUVAGE, C. -1961-Recherches géobotaniques sur les subéraies marocaines. Rabat.

WILLKOMM, H.M. y J.M.CH. LANGE -1880Prodromus florae hispanicae, III. Stuttgart.

Aceptado para su publicación en Julio de 1996

Dirección de los autores. I. Sánchez García: Parque de Capuchinos, Blq. 3-2 D. E-11405 Jerez de la Frontera (Cádiz) y A. Galán de Mera: Departamento de Biología. Laboratorio de Botánica. Universidad San Pablo-CEU. Ap. 67. E-28660 Boadilla del Monte (Madrid). Fax. (91) 3510475. 